\title{
Identification and Monitoring of International Travelers During the Initial Phase of an Outbreak of COVID-19 - California, February 3-March 17, 2020
}

Jennifer F. Myers, $\mathrm{MPH}^{1}$; Robert E. Snyder, $\mathrm{PhD}^{1}$; Charsey Cole Porse, $\mathrm{PhD}^{1}$; Selam Tecle, $\mathrm{MPH}^{1}$; Phil Lowenthal, $\mathrm{MPH}^{1}$; Mary E. Danforth, PhD ${ }^{1}$; Edward Powers, DVM ${ }^{1}$; Amanda Kamali, MD ${ }^{1}$; Seema Jain, MD ${ }^{1}$; Curtis L. Fritz, DVM, PhD ${ }^{1}$; Shua J. Chai, MD ${ }^{1,2}$; Traveler Monitoring Team

\section{On May 11, 2020, this report was posted as an MMWR Early Release on the MMWR website (https://www.cdc.gov/mmwr).}

The threat of introduction of coronavirus disease 2019 (COVID-19) into the United States with the potential for community transmission prompted U.S. federal officials in February 2020 to screen travelers from China, and later Iran, and collect and transmit their demographic and contact information to states for follow-up. During February 5-March 17, 2020, the California Department of Public Health (CDPH) received and transmitted contact information for 11,574 international travelers to 51 of 61 local health jurisdictions at a cost of 1,694 hours of $\mathrm{CDPH}$ personnel time. If resources permitted, local health jurisdictions contacted travelers, interviewed them, and oversaw 14 days of quarantine, self-monitoring, or both, based on CDC risk assessment criteria for COVID-19. Challenges encountered during follow-up included errors in the recording of contact information and variation in the availability of resources in local health jurisdictions to address the substantial workload. Among COVID-19 patients reported to CDPH, three matched persons previously reported as travelers to CDPH. Despite intensive effort, the traveler screening system did not effectively prevent introduction of COVID-19 into California. Effectiveness of COVID-19 screening and monitoring in travelers to California was limited by incomplete traveler information received by federal officials and transmitted to states, the number of travelers needing follow-up, and the potential for presymptomatic and asymptomatic transmission. More efficient methods of collecting and transmitting passenger data, including electronic provision of flight manifests by airlines to federal officials and flexible text-messaging tools, would help local health jurisdictions reach out to all at-risk travelers quickly, thereby facilitating timely testing, case identification, and contact investigations. State and local health departments should weigh the resources needed to implement incoming traveler monitoring against community mitigation activities, understanding that the priorities of each might shift during the COVID-19 pandemic.

On December 31, 2019, Chinese authorities reported detection of a novel coronavirus (SARS-CoV-2) among persons with pneumonia in Wuhan City, in Hubei Province; the disease was subsequently named COVID-19 (1). The threat of importation of SARS-CoV-2 from China into the United States prompted the executive order limiting travel from China on January 31, 2020, and implemented starting February 3, 2020.
U.S. citizens and lawful permanent residents and their families who had been in China in the past 14 days were allowed to enter the United States. To facilitate screening of these persons upon arrival in the United States, the U.S. Department of Homeland Security directed all flights from China to 11 U.S. airports starting on February 3.* Customs and Border Protection agents interviewed travelers arriving from China regarding signs and symptoms compatible with COVID- $19^{\dagger}$ and, working with the Department of Homeland Security and CDC, collected traveler demographic and contact information and provided travelers with instructions for self-monitoring. CDC oversaw secondary screening of symptomatic travelers. Customs and Border Protection transmitted demographic and contact information for all arriving travelers to CDC, regardless of symptom status at the time of arrival, which forwarded this information securely to state public health authorities for follow-up through CDC's Epi-X network. ${ }^{\S}$ Initially, CDC transmitted traveler information to $\mathrm{CDPH}$ only for persons arriving on flights from China; however, on March 5, travelers who had been to Iran within the preceding 14 days were added to these notices following CDC's recommendation to avoid nonessential travel to Iran (2). State and local public health officials were requested, if resources permitted, to contact travelers, interview them to ascertain signs or symptoms of illness and additional risk exposures, and oversee 14 days of quarantine, self-monitoring, or both, based on CDC risk assessment criteria for COVID-19. This report summarizes CDPH's experience with the traveler monitoring program for COVID-19 among travelers from China and Iran who had traveler contact information sent to CDPH by CDC.

Beginning February 3, CDPH redirected public health medical officers, epidemiologists, and other personnel to the CDPH Return Traveler Monitoring team. From February 5, when $\mathrm{CDPH}$ first received CDC traveler notifications, through the decommission of CDPH's traveler monitoring program on March 17, CDPH processed 2,266 Epi-X notifications of arriving travelers, representing 12,061 individual travelers (Figure). CDPH processed a median of 39 notifications per

\footnotetext{
* Notice of arrival restrictions applicable to flights carrying persons who have recently traveled from or were otherwise present within the People's Republic of China. 19 C.F.R. Chap. 1; 49 C.F.R. Chap. 12 (2020). https://www.dhs. gov/sites/default/files/publications/20_0202_dhs-arrival-restriction-frn-2.pdf. $\dagger$ Fever, cough, shortness of breath, or who appeared visibly ill.

${ }^{\$}$ https://emergency.cdc.gov/epix/index.asp.
} 


\section{Summary}

What is already known about this topic?

To reduce introductions of COVID-19 into the United States, travelers from selected countries were screened upon entry, and their contact information forwarded to states for monitoring.

What is added by this report?

During February 3-March 17, 2020, California received, corrected, and transmitted information on 11,574 travelers to local health jurisdictions for follow-up. Three travelers were matched to three of the 26,182 patients with COVID-19 reported to California by April 15 .

What are the implications for public health practice?

Monitoring travelers was labor-intensive and limited by incomplete information, volume of travelers, and potential for asymptomatic transmission. Health departments need to weigh the resources needed for monitoring against those needed for implementing mitigation activities during the COVID-19 pandemic.

day (range $=1-146$ ), with a median of 23 individual records per notification (range $=1-250$ ), equating to a median of 1,431 travelers per week.

Before sending to local health jurisdictions, CDPH staff members reviewed individual records to identify the destination jurisdiction for each traveler and any possible demographic and contact information errors. Among 1,523 (13\%) records with one or more identifiable errors, 1,135 (75\%) did not have a correct U.S.-based telephone number, 603 (40\%) were duplicate records, and 487 (32\%) had insufficient location data or the traveler resided outside of California. Additional suspected errors in reported names and dates of birth were noted, including likely name misspellings and out-of-range dates of birth; flight manifests or other independent records to verify traveler information were unavailable. Following resolution of identifiable and correctable errors, 11,574 (96\%) records were assigned and sent to 51 of California's 61 local health jurisdictions. The number of travelers in each affected jurisdiction ranged from one to 4,852. Among the 11,574 California travelers processed by $\mathrm{CDPH}$, three were matched by name and date of birth to three of 26,182 confirmed COVID-19 cases in California reported to CDPH through the California reportable disease system as of April 15. Two of these COVID-19 patients had traveled from Iran and were tested several days after arrival, and their cases were laboratoryconfirmed. A third patient had traveled from China but was tested on March 30, approximately 6 weeks after returning to the United States and after the date local health jurisdictions would have ended follow-up.
During the 7-week period of the program, CDPH staff members devoted an estimated 1,694 total person-hours (equivalent to six employees working full-time for 7 weeks) processing traveler Epi-X notices and assigning travelers to local health jurisdictions; 576 (34\%) of these person-hours occurred outside regular working hours. The additional personnel time incurred in the 51 affected California local health jurisdictions for follow-up was not available; the capacity for local health jurisdictions to conduct follow-up varied considerably according to resources and traveler volume.

\section{Discussion}

Airport entry screening and quarantine and monitoring of travelers can be an effective tool for preventing and slowing importation of some diseases into the United States (3). To be effective, it requires accurate contact information for travelers. Substantial time was devoted to addressing incorrect traveler contact information at $\mathrm{CDPH}$, and later at the local jurisdiction level, which compromised timely contact of travelers or completely precluded reaching some travelers. More efficient methods of collecting passenger data, including electronic provision of flight manifests by airlines to federal officials to transmit to states, would help local health jurisdictions quickly reach out to all travelers at risk, thereby facilitating timely testing, case identification, and contact investigations. Flexible electronic messaging platforms, such as text messaging, and additional personnel resources for local health jurisdictions with limited capacity for follow-up of travelers could have further increased the likelihood of case identification.

During previous international disease outbreaks, screening and quarantine or monitoring of travelers was most effective when infected travelers could be readily identified and when they arrived in numbers that could be tracked using available public health resources (3). For example, traveler monitoring in California for Ebola virus disease (Ebola) from Africa during 2014-2015 was effective because Ebola has obvious clinical manifestations, is contagious only after symptom onset, and a smaller number of travelers required monitoring. A median of 21 travelers per week from three Ebola-affected countries in Africa were monitored in California over 17 months (CDPH, unpublished data, 2015), compared with the median of 1,431 travelers monitored per week for COVID-19 over 7 weeks.

The benefits of screening for case detection at the airport might be limited for a respiratory disease with the potential for presymptomatic and asymptomatic transmission, such as COVID-19 (4). Monitoring travelers after they have cleared screening at the airport can be valuable for certain diseases such as Ebola, but it is labor-intensive for public health officials. Effectiveness of traveler monitoring can vary by pandemic phase (5) and is likely more effective during the 
FIGURE. Arriving international travelers monitored for COVID-19 ( $N=12,061)$, by country of travel origin and cases identified* — California, February 5-March 17, 2020

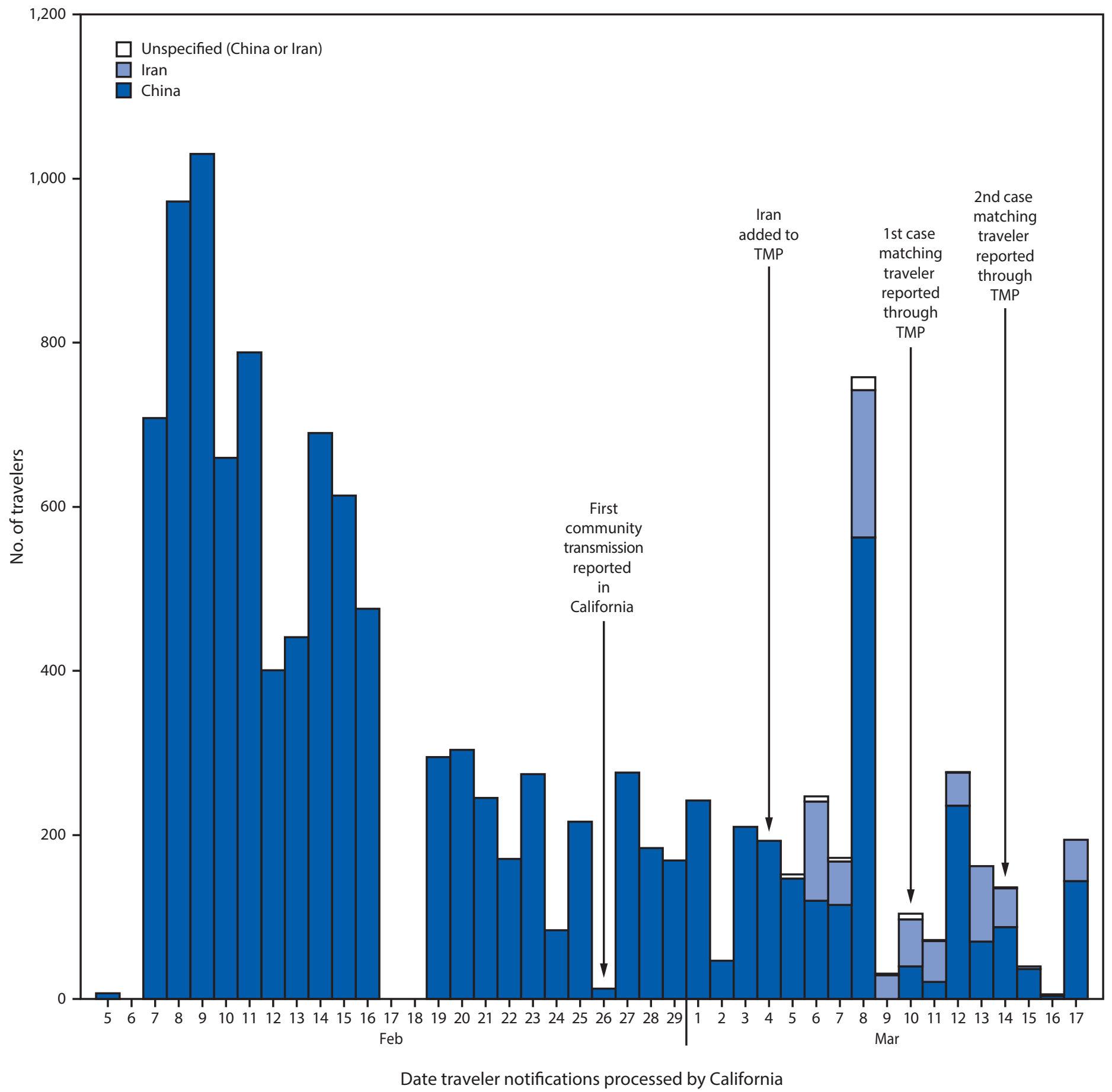

Abbreviations: $\mathrm{CDPH}=$ California Department of Public Health; COVID-19 = coronavirus disease 2019; TMP = traveler monitoring program.

* The first and second cases identified occurred in travelers arriving from Iran and were laboratory-confirmed; these travelers were reported by CDC to CDPH and matched by traveler's date of birth and name to two confirmed COVID-19 cases reported to CDPH through the California reportable disease system as of April 15, 2020. 
initial containment phase, when the focus is on reducing the number of new introductions and delaying the onset of community transmission.

The findings in this report are subject to at least three limitations. First, arriving travelers who were infected but asymptomatic, whether screened upon arrival or monitored after arrival by their destination's local health jurisdiction, would not have been tested and reported as a case to CDPH. Similarly, infected travelers who became symptomatic after screening would have needed to seek care and be tested to be reported as a case to CDPH. Second, errors in names and dates of birth collected from travelers limited CDPH's ability to match travelers to reported COVID-19 cases in California, potentially underestimating the number of travelers that could be matched to a case. Third, as community transmission of COVID-19 became more widespread across California, determining whether travel versus community transmission resulted in infection became less certain. Concurrently, fewer data were collected on individual cases as case numbers increased, making assessment of traveler monitoring effectiveness more difficult. These limitations might have contributed to the similar national findings of 14 confirmed COVID-19 cases identified among the approximately 268,000 travelers screened as of April 21, 2020 (G).

Despite intensive effort, the traveler screening system did not effectively prevent introduction of COVID-19 into California. Incomplete traveler information received by federal officials and transmitted to states, the number of travelers requiring follow-up, and the potential for presymptomatic and asymptomatic transmission likely contributed to onset of community transmission and the need to shift to mitigation measures. In California, the first confirmed case of COVID-19 without known exposure to a traveler or a patient with COVID-19 was reported to CDPH on February 26, 2020. Once community transmission was documented in several California counties, local health jurisdictions needed to weigh the effectiveness and costs of continued traveler monitoring for imported disease against implementation of mitigation measures to slow local disease transmission and allow health care systems to prepare for increased caseloads. Multiple California counties declared shelter-in-place orders on March 16; CDPH discontinued the traveler monitoring program on March 17. In later phases of the pandemic, as community transmission decreases following successful mitigation measures, containment strategies such as reconfigured and focused traveler monitoring, with accurate traveler demographic and contact information and increased staffing capacity in public health agencies, might be useful to maintain low disease incidence if there are subsequent disease waves.

\section{Acknowledgments}

California's 51 local health jurisdictions that conducted follow-up on arriving travelers.

\section{Traveler Monitoring Team}

Duc Vugia, California Department of Public Health; James P. Watt, California Department of Public Health; Clive Brown, CDC.

Corresponding author: Shua J. Chai, shua.chai@cdph.ca.gov, 510-412-4679.

${ }^{1}$ Division of Communicable Disease Control, California Department of Public Health; ${ }^{2}$ Career Epidemiology Field Officer, Center for Preparedness and Response, CDC.

All authors have completed and submitted the International Committee of Medical Journal Editors form for disclosure of potential conflicts of interest. No potential conflicts of interest were disclosed.

\section{References}

1. World Health Organization. Novel coronavirus (2019-nCoV) situation report - 1. Geneva, Switzerland: World Health Organization; 2020. https://www.who.int/docs/default-source/coronaviruse/situationreports/20200121-sitrep-1-2019-ncov.pdf?sfvrsn=20a99c10_4

2. Office of the President of the United States. Proclamation on suspension of entry as immigrants and nonimmigrants of persons who pose a risk of transmitting 2019 novel coronavirus. Washington, DC: Office of the President of the United States; 2020. https://www.whitehouse. gov/presidential-actions/proclamation-suspension-entry-immigrantsnonimmigrants-certain-additional-persons-pose-risk-transmittingcoronavirus/

3. Fritz CL, Dennis DT, Tipple MA, Campbell GL, McCance CR, Gubler DJ. Surveillance for pneumonic plague in the United States during an international emergency: a model for control of imported emerging diseases. Emerg Infect Dis 1996;2:30-6. https://doi.org/10.3201/ eid0201.960103

4. Arons MM, Hatfield KM, Reddy SC, et al. Presymptomatic SARS-CoV-2 infections and transmission in a skilled nursing facility. N Engl J Med 2020. Epub March 24, 2020. https://doi.org/10.1056/NEJMoa2008457

5. World Health Organization. Key considerations for repatriation and quarantine of travellers in relation to the outbreak of novel coronavirus 2019-nCoV. Geneva, Switzerland: World Health Organization; 2020. https://www.who.int/news-room/articles-detail/key-considerations-forrepatriation-and-quarantine-of-travellers-in-relation-to-the-outbreak-ofnovel-coronavirus-2019-ncov\#.XpMvFUk_bhc.ema

6. Schuchat A; CDC COVID-19 Response Team. Public health response to the initiation and spread of pandemic COVID-19 in the United States, February 24-April 21, 2020. MMWR Morb Mortal Wkly Rep 2020;69:551-6. http://dx.doi.org/10.15585/mmwr.mm6918e2 